Research Article

\title{
A Mathematical Model for Nipah Virus Infection
}

\author{
Assefa Denekew Zewdie $\mathbb{D}^{1,2}$ and Sunita Gakkhar ${ }^{1}$ \\ ${ }^{1}$ Department of Mathematics, Indian Institute of Technology Roorkee, Roorkee, 247667 Uttarakhand, India \\ ${ }^{2}$ Department of Mathematics, Debre Tabor University, Debre Tabor, Ethiopia
}

Correspondence should be addressed to Assefa Denekew Zewdie; assefadagi@gmail.com

Received 11 June 2020; Accepted 16 July 2020; Published 30 September 2020

Academic Editor: Ferenc Hartung

Copyright (c) 2020 Assefa Denekew Zewdie and Sunita Gakkhar. This is an open access article distributed under the Creative Commons Attribution License, which permits unrestricted use, distribution, and reproduction in any medium, provided the original work is properly cited.

\begin{abstract}
It has been reported that unprotected contact with the dead bodies of infected individuals is a plausible way of Nipah virus transmission. An SIRD model is proposed in this paper to investigate the impact of unprotected contact with dead bodies of infected individuals before burial or cremation and their disposal rate on the dynamics of Nipah virus infection. The model is analyzed, and the reproduction number is computed. It is established that the disease-free state is globally asymptotically stable when the reproduction number is less than unity and unstable if it is greater than unity. By using the central manifold theory, we observe that the endemic equilibrium is locally stable near to unity. It is concluded that minimizing unsafe contact with the infected dead body and/or burial or cremation as fast as possible contributes positively. Further, the numerical simulations for the given choice of data and initial conditions illustrate that the endemic state is stable and the disease persists in the community when the reproduction number is greater than one.
\end{abstract}

\section{Introduction}

Nipah virus is first identified in the Malaysian village of Sungai Nipah during an outbreak of encephalitis and respiratory illness. Pig farmers got virus from pigs. There were more than 265 cases including 105 deaths from September 1998 to April 1999 [1-3]. Later on, it spreads to other south-east Asian regions. It is a zoonotic disease, transmitted from animals to humans caused by a Nipah virus which belongs to the Paramyxoviridae family. It causes a severe illness such as brain inflammations or respiratory infection in animal and human population followed by death. The disease is highly infectious and spreads through the community with infected animals; however, no prophylaxis or effective treatment is available. In the absence of effective drugs, its treatment is limited to a symptomatic treatment, hospitalization, and quarantine. Educating people and creating awareness is the only way to control the spread of infection due to nonavailability of any vaccine for Nipah virus [4].

The natural reservoir of Nipah virus is fruit bats belonging to the Pteropus genus in Pteropodidae family [5]. Over 50 species of Pteropus bats are inhabiting the south and south-east Asian regions [4]. Many different pathways are observed for the Nipah virus transmission to human: from fruit bats to human, from fruit bats to animal then to human, and from human to human $[2,6]$. Consumption of date palm sap is popular in countries of the south-east Asian region [4], and it is commonly collected in the cold season particularly in rural areas of Bangladesh [7]. Fruit bats also consume and contaminate the date palm and its sap with their secretions, e.g., saliva and urine. Human consumption of fruits or fruit products was one of the transmission route of Nipah from fruit bats to human in Bangladesh $[6,8]$. The pigs will get infected by consuming dropped fruits that are partially eaten and infected by bats. These infected pigs transmit Nipah virus to human through fluid contact, while handling ill pigs or through consumption of pork [2]. In Malaysia and Singapore, most Nipah infection cases were observed in individuals having frequent contact with sick pigs $[9,10]$.

Further, person to person transmission was observed during the Bangladesh Nipah outbreak of the year 2004 [11, 12]. In the outbreaks of Siliguri, India, the Nipah virus was 
not found in samples obtained from animals. However, samples of human fluid secretions found in health care settings were positive for Nipah virus. In particular, healthcare workers, family care givers, and hospital visitors who had exposure to secretions of Nipah virus patients with direct contact or through contaminated towels, bed sheets, etc., got infected $[13,14]$.

Some infectious diseases, like Ebola virus disease [15], can transmit from person to person if there is unsafe direct contact with the dead body of infected individuals during funeral ceremonies or last rites. Unprotected contact of corpse of an infected individual before burial/cremation is another way of transmission of the Nipah virus from human to human $[16,17]$.

Many researchers have studied the pathology and epidemiology of the Nipah virus disease, but very few models are available for it and presented as follows. Biswas studied the disease dynamics using SIR basic mathematical model [18]. $\mathrm{He}$ further investigated this model and studied the possible control and preventive strategies through optimal control $[19,20]$. Optimal control is also carried out in another SIR model [21]. Mondal et al. have proposed an SEIR model to study the dynamics of the disease by incorporating two control parameters (number of quarantined individuals and enhanced personal hygiene) which have not yet used before in a Nipah dynamic model [22]. Shah et al. proposed an SEI model considering bat to human and human to human disease transmission [23]. The unprotected contact of the dead body of the Nipah virus-infected person is one way of transmitting disease from human to human which has not been considered so far. Durgesh et al. in [24] proposed an SVEIR model by considering bat-human interaction, and they analyzed that vaccination has its own role for controlling the disease to spread. Nita et al. also proposed an SEIHD epidemic model with bat-human interaction in [25] and incorporated control measures such as spray insecticides, buried bats, self-prevention, and hospitalization. They also analyzed the dynamics of the disease and optimal control. Agarwal and Singh in [26] proposed an SEI epidemic model for flying foxes and human. They consider a virus compartment incorporating the fractional order differential equation to analyze. Keeping this in view, an SIRD model is proposed and investigated by incorporating unsafe burial or unprotected contact of dead bodies of infected individuals capable of spreading Nipah virus and its disposal rate. The objective of this study is to investigate the role of safe burial or cremation of dead bodies in the control of Nipah virus. This paper is organized according to the following: Section 2 presents the formulation of the proposed model and description of the parameters. In Section 3, the model analysis including the basic reproduction number, equilibrium points, and their stabilities are presented. In Section 4, the numerical simulation is presented and the graphical results are discussed. Finally, the conclusion is presented in Section 5.

\section{Model Formulation}

The total population $N(t)$ is divided into three mutually disjoint compartments, namely, susceptible $S(t)$, infected $I(t)$,

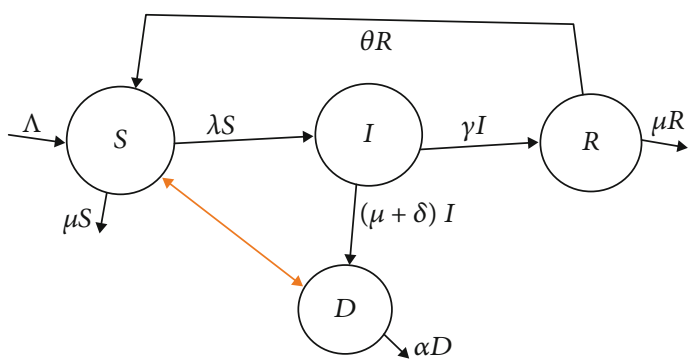

Figure 1: Flow chart of the Nipah model.

and recovered $R(t)$ such that $N=S+I+R$. The susceptible are those individuals who are not infected, but they will become infected when they come in contact with infectious individuals. Infected are those individuals who are infectious and may infect others. Recovered are individuals who have recovered from infectiousness either with treatment or by their own. Moreover, the dead bodies of Nipah-infected individuals can also transmit the disease. This model incorporates deceased body compartment $D(t)$; it represents the number of unburied dead bodies of infected individuals. The natural death of susceptible and recovered is excluded from $D$ compartment. The constant parameters $\Lambda$ and $\mu$ are the natural birth and death rates, respectively, while $\theta$ is the rate of loss of temporary immunity acquired by recovered individuals. Considering the parameter $\beta_{1}$ to be the effective unprotected contact rate of susceptible individuals to get infection from dead bodies of Nipah-infected individuals and $\beta_{2}$ to be the effective contact rate of susceptible individuals to get infection from infected individuals, then the force of infection representing the effective transmission rate $\lambda$ is given by

$$
\lambda=\frac{\left(\kappa \beta_{1} D+\beta_{2} I\right)}{N} .
$$

Parameter $\kappa$ represents a constant fraction of unsafe or unprotected handling of dead bodies leading to the spread of Nipah infection. Further, there is no possibility that susceptible individuals can get infection from the dead body of infected individuals when $\kappa=0$. The susceptible class is increased by $\Lambda$ and $\theta$ rates and decreased by $\lambda$ and $\mu$ rates. The infected compartment is increased by $\lambda$ and decreased by $\gamma, \mu$, and $\delta$ rates due to developing immunity, natural death, and disease-induced death, respectively. The recovered compartment is increased by $\gamma$ rate and decreased by $\mu$ and $\theta$ rates. The deceased body compartment is increased by $\mu$ and $\delta$ rates released from infected class and buried with $\alpha$ rate. From those that are dead, $\kappa$ rates of them can transfer the disease before burial due to unprotected contact. This model is described by the flow diagram given in (Figure 1) and nonlinear ordinary differential equations given in (2). The description and values of parameters used in the model are given in (Table 1). 
TABLE 1: Description and values of parameters.

\begin{tabular}{|c|c|c|c|}
\hline Parameter & Description & Parameter values & Source \\
\hline$\Lambda$ & Recruitment rate to susceptible class & 6102 day $^{-1}$ & {$[22]$} \\
\hline$\beta_{1}$ & Rate of infection from dead bodies of infected individuals & 0.65 day $^{-1}$ & Assumed \\
\hline$\beta_{2}$ & Rate of infection from infected individuals & 0.75 day $^{-1}$ & {$[22]$} \\
\hline$\theta$ & Rate of loss of immunity & 0.85 day $^{-1}$ & Assumed \\
\hline$\gamma$ & Rate of recovered individuals from infected class & 0.09 day $^{-1}$ & {$[22]$} \\
\hline$\alpha$ & Rate of disposition (burial/cremation) of dead bodies & Variable & Assumed \\
\hline$\mu$ & Natural death rate & 0.000038642 day $^{-1}$ & {$[22]$} \\
\hline$\delta$ & Rate of disease-induced death & 0.76 day $^{-1}$ & {$[22]$} \\
\hline$\kappa$ & Fraction of dead bodies that are not handled safely & {$[0,1]$} & Assumed \\
\hline
\end{tabular}

$$
\begin{aligned}
& \frac{d S}{d t}=\Lambda-\lambda S-\mu S+\theta R \equiv f_{1}(S, I, R, D, t), \\
& \frac{d I}{d t}=\lambda S-(\mu+\delta+\gamma) I \equiv f_{2}(S, I, R, D, t), \\
& \frac{d R}{d t}=\gamma I-(\mu+\theta) R \equiv f_{3}(S, I, R, D, t), \\
& \frac{d D}{d t}=(\mu+\delta) I-\alpha D \equiv f_{4}(S, I, R, D, t) .
\end{aligned}
$$

The following nonnegative initial conditions are associated with the dynamical system given in the model (2):

$$
\begin{gathered}
S(0)=S_{0}>0, \\
I(0)=I_{0} \geq 0, \\
R(0)=R_{0} \geq 0, \\
D(0)=D_{0} \geq 0 .
\end{gathered}
$$

\section{Model Analysis}

It can be easily show that the functions $f_{i}, i=1,2,3,4$ are sufficiently smooth and satisfy the Lipchitz condition in $R_{+}^{4}$. Accordingly, the IVP in the system (2) admits a unique solution in $R_{+}^{4}$.

\subsection{Invariant Region and Positivity}

Lemma 1. The system in (2) with initial conditions given in (3) has a positive invariant solution in the region $\Omega=\left\{(S, I, R, D) \in R_{+}^{4}: N=S+I+R \leq(\Lambda / \mu), D \leq((\Lambda\right.$ $(\mu+\delta)) / \alpha \mu)\}$.

Proof. All existing solutions starting from nonnegative initial conditions remain nonnegative for all time $t \geq 0$. It can be shown by contradiction [27]. Let there exists a time $t_{1}>0$ such that

$$
\begin{aligned}
S\left(t_{1}\right) & =0, \\
S(t) & \geq 0, \\
I(t) & \geq 0, \\
R(t) & \geq 0, \\
D(t) & \geq 0, \\
0 & \leq t \leq t_{1} .
\end{aligned}
$$

There exists $t_{2}>0$ such that

$$
\begin{aligned}
I\left(t_{2}\right) & =0, \\
S(t) & \geq 0, \\
I(t) & \geq 0, \\
R(t) & \geq 0, \\
D(t) & \geq 0, \\
0 & \leq t \leq t_{2} .
\end{aligned}
$$

There exists a $t_{3}>0$ such that

$$
\begin{aligned}
R\left(t_{3}\right) & =0, \\
S(t) & \geq 0, \\
I(t) & \geq 0, \\
R(t) & \geq 0, \\
D(t) & \geq 0, \\
0 & \leq t \leq t_{3} .
\end{aligned}
$$

There exists $t_{4}>0$ such that

$$
\begin{aligned}
D\left(t_{4}\right) & =0, \\
S(t) & \geq 0, \\
I(t) & \geq 0, \\
R(t) & \geq 0, \\
D(t) & \geq 0, \\
0 & \leq t \leq t_{4} .
\end{aligned}
$$


From the first equation of the system in model (2) and from case (4), we have

$$
S^{\prime}\left(t_{1}\right)=\Lambda-\lambda S\left(t_{1}\right)-\mu S\left(t_{1}\right)+\theta R\left(t_{1}\right)=\Lambda+\theta R\left(t_{1}\right)>0 .
$$

This means $S(t)<0$, which contradicts the fact that $S(t)$ is initially nonnegative; it implies $S(t)$ is positive. Similarly, one can show that the solution of all state variables is positive.

The total population of individuals is given by $N=$ $S+I+R$ and adding equations of the system in model (2), we have

$$
\frac{d N(t)}{d t}=\Lambda-\mu N(t)-\delta I(t) \leq \Lambda-\mu N(t) .
$$

It follows that:

$$
N(t) \leq \frac{\Lambda}{\mu}-\left(\frac{\Lambda}{\mu}-N_{0}\right) e^{-\mu t},
$$

where $N(0)=N_{0}$ is the initial value of the total population and as $t \longrightarrow \infty, N(t) \leq(\Lambda / \mu)$ and

$$
\frac{d D(t)}{d t}=(\mu+\delta) I(t)-\alpha D(t)
$$
$t \geq 0$.

But $N(t)=S(t)+I(t)+R(t) \leq(\Lambda / \mu) \Rightarrow I(t) \leq(\Lambda / \mu)$ as

It follows that

$$
\begin{aligned}
\frac{d D(t)}{d t} & =(\mu+\delta) I(t)-\alpha D(t) \Rightarrow D(t) \\
& \leq \frac{\Lambda(\mu+\delta)}{\alpha \mu}-\left(\frac{\Lambda(\mu+\delta)}{\alpha \mu}-D_{0}\right) e^{-\alpha t},
\end{aligned}
$$

and as $t \longrightarrow \infty, D(t) \longrightarrow((\Lambda(\mu+\delta)) / \alpha \mu)$.

Hence, the region $\Omega$ is positively invariant for the system (2) and it attracts all solutions of the equations in the system.

3.2. Reproduction Number. In the absence of the disease, the system given in model (2) has an equilibrium point $E^{0}=((\Lambda / \mu), 0,0,0)$. The infectious class is $X=(I, D)^{T}$, and the rate of appearance of new infection in each infectious class is denoted by $\mathscr{F}(X)$ and given by

$$
\mathscr{F}(x)=\left(\frac{\left(\kappa \beta_{1} D+\beta_{2} I\right) S}{N}, 0\right)^{T} .
$$

The rate of other transition between infectious classes is denoted by $\mathscr{V}(X)$ and given by

$$
\mathscr{V}(X)=((\gamma+\mu+\delta) \mathrm{I},-(\mu+\delta) I+(\alpha D))^{T},
$$

where

$$
\begin{aligned}
\mathscr{f}(\mathscr{F}) & =\left(\begin{array}{ll}
\frac{\partial \mathscr{F}_{1}}{\partial I} & \frac{\partial \mathscr{F}_{1}}{\partial D} \\
\frac{\partial \mathscr{F}_{2}}{\partial I} & \frac{\partial \mathscr{F}_{2}}{\partial D}
\end{array}\right) \\
& =\left(\begin{array}{cc}
\frac{\beta_{2} S N-\left(\kappa \beta_{1} D+\beta_{2} I\right) S}{N^{2}} & \frac{\kappa \beta_{1} S N-\left(\kappa \beta_{1} D+\beta_{2} I\right) S}{N^{2}} \\
0 & 0
\end{array}\right), \\
\mathscr{J}(\mathscr{V}) & =\left(\begin{array}{cc}
\frac{\partial \mathscr{V}_{1}}{\partial I} & \frac{\partial \mathscr{V}_{1}}{\partial D} \\
\frac{\partial \mathscr{V}_{2}}{\partial I} & \frac{\partial \mathscr{V}_{2}}{\partial D}
\end{array}\right)=\left(\begin{array}{cc}
\gamma+\mu+\delta & 0 \\
-(\mu+\delta) & \alpha
\end{array}\right) .
\end{aligned}
$$

At disease-free equilibrium point $\left(E^{0}\right)$, the matrices $\mathscr{f}(\mathscr{F})$ and $\mathscr{f}(\mathscr{V})$ will give us

$$
\begin{aligned}
\left.\mathcal{J}(\mathcal{F})\right|_{E^{0}} & =F=\left(\begin{array}{cc}
\beta_{2} & \kappa \beta_{1} \\
0 & 0
\end{array}\right), \\
\left.\Rightarrow \mathcal{J}(\mathcal{V})\right|_{E^{0}} & =V=\left(\begin{array}{cc}
\gamma+\mu+\delta & 0 \\
-(\mu+\delta) & \alpha
\end{array}\right), \\
V^{-1} & =\left(\begin{array}{cc}
\frac{1}{\gamma+\mu+\delta} & 0 \\
\frac{\mu+\delta}{\alpha(\gamma+\mu+\delta)} & \frac{1}{\alpha}
\end{array}\right), \\
\Rightarrow F V^{-1} & =\left(\begin{array}{cc}
\frac{\kappa(\delta+\mu) \beta_{1}+\alpha \beta_{2}}{\alpha(\gamma+\mu+\delta)} & \frac{\kappa \beta_{1}}{\alpha} \\
0 & 0
\end{array}\right) .
\end{aligned}
$$

Now the spectral radius $\left(\rho\left(F V^{-1}\right)\right)$ of the matrix $F V^{-1}$ is given by

$$
\rho\left(F V^{-1}\right)=\frac{\kappa(\delta+\mu) \beta_{1}+\alpha \beta_{2}}{\alpha(\gamma+\mu+\delta)} .
$$

Applying next-generation matrix approach [28], the reproduction number $\left(R_{D}\right)$ is computed as

$$
R_{D}=\frac{\kappa(\delta+\mu) \beta_{1}+\alpha \beta_{2}}{\alpha(\gamma+\mu+\delta)}=R_{0}+\frac{\kappa(\delta+\mu) \beta_{1}}{\alpha(\gamma+\mu+\delta)},
$$

where $R_{0}$ is the basic reproduction number of the SIR model when $\kappa=0$; that is, all infected dead bodies are safely buried. Further, it may be noted that $R_{D}$ increases as $\kappa$ increases and it has an inverse relationship with $\alpha$; that means the quicker the dead bodies are buried or cremated, the smaller will be the reproduction number.

3.3. Existence of Equilibrium Point. The system given in model (2) always admits a disease-free equilibrium $E^{0}=(\Lambda /$ $\mu, 0,0,0)$. Further, let the $\mu$ system have an endemic 
equilibrium point, denoted by $E^{*}=\left(S^{*}, I^{*}, R^{*}, D^{*}\right)$. Then, the force of infection from equation (1) at this equilibrium point is

$$
\begin{aligned}
& \lambda^{*}=\frac{\kappa \beta_{1} D^{*}+\beta_{2} I^{*}}{N^{*}}, \\
& N^{*}=S^{*}+I^{*}+R^{*} .
\end{aligned}
$$

Substituting and simplifying yields

$$
\begin{aligned}
\lambda^{*}+a \lambda^{*} & =0 \Rightarrow \lambda^{*}=0, \\
\lambda^{*} & =-a,
\end{aligned}
$$

where

$$
\begin{aligned}
a & =\frac{(\theta+\mu)\left(\alpha(\gamma+\mu+\delta)-\left(\kappa(\delta+\mu) \beta_{1}+\alpha+\beta_{2}\right)\right)}{\alpha(\mu+\theta+\gamma)} \\
& =\frac{(\theta+\mu)(\gamma+\mu+\delta)}{(\mu+\theta+\gamma)}\left(1-R_{D}\right) .
\end{aligned}
$$

For $R_{D}<1$ equation (21) has only one nonnegative solution $\lambda^{*}=0$, which is a disease-free equilibrium. But it has two nonnegative solutions, one is the disease free and the other is endemic equilibrium for $R_{D}>1$. This result gives us the endemic equilibrium point exists in the system of model (2) and the following lemma.

Lemma 2. For $R_{D}>1$, the system in model (2) has a unique endemic equilibrium point $\left(E^{*}\right)$ given by, $E^{*}=\left(S^{*}, I^{*}, R^{*}\right.$, $D^{*}$ ), where

$$
\begin{aligned}
S^{*} & =\frac{\Lambda(\gamma+\mu+\theta)}{\delta(\theta+\mu)\left(R_{D}-1\right)+\mu(\gamma+\theta+\mu) R_{D}}, \\
I^{*} & =\frac{\Lambda(\theta+\mu)\left(R_{D}-1\right)}{\delta(\theta+\mu)\left(R_{D}-1\right)+\mu(\gamma+\theta+\mu) R_{D}}, \\
R^{*} & =\frac{\Lambda \gamma\left(R_{D}-1\right)}{\delta(\theta+\mu)\left(R_{D}-1\right)+\mu(\gamma+\theta+\mu) R_{D}}, \\
D^{*} & =\frac{\Lambda(\delta+\mu)(\theta+\mu)\left(R_{0}-1\right)}{\alpha\left(\delta(\theta+\mu)\left(R_{D}-1\right)+\mu(\gamma+\theta+\mu) R_{D}\right)} .
\end{aligned}
$$

It is noted that the endemic point reduces to disease-free equilibrium point for $R_{D}=1$.

\subsection{Stability Analysis}

\subsubsection{Local Stability of Equilibrium Points}

Proposition 1. The disease-free equilibrium point of the system given in model (2) is locally asymptotically stable if $R_{D}<1$ and unstable if $R_{D}>1$.
Proof. The Jacobian matrix of system (2) is

$$
J(X)=\left(\begin{array}{llll}
\frac{\partial f_{1}}{\partial S} & \frac{\partial f_{1}}{\partial I} & \frac{\partial f_{1}}{\partial R} & \frac{\partial f_{1}}{\partial D} \\
\frac{\partial f_{2}}{\partial S} & \frac{\partial f_{2}}{\partial I} & \frac{\partial f_{2}}{\partial R} & \frac{\partial f_{2}}{\partial D} \\
\frac{\partial f_{3}}{\partial S} & \frac{\partial f_{3}}{\partial I} & \frac{\partial f_{3}}{\partial R} & \frac{\partial f_{3}}{\partial D} \\
\frac{\partial f_{4}}{\partial S} & \frac{\partial f_{4}}{\partial I} & \frac{\partial f_{4}}{\partial R} & \frac{\partial f_{4}}{\partial D}
\end{array}\right),
$$

where $X=(S, I, R, D)^{T}$.

At disease-free equilibrium point, the Jacobian matrix gives

$$
J\left(E^{0}\right)=\left(\begin{array}{cccc}
-\mu & -\beta_{2} & \theta & -\kappa \beta_{1} \\
0 & \beta_{2}-(\gamma+\mu+\delta) & 0 & \kappa \beta_{1} \\
0 & \gamma & -(\theta+\mu) & 0 \\
0 & \delta+\mu & 0 & -\alpha
\end{array}\right) .
$$

The Jacobian matrix (24) of the system in (2) at the disease-free equilibrium point gives the following polynomial characteristic equation:

$$
(\lambda+\mu)(\lambda+(\theta+\mu))\left(\lambda^{2}+B \lambda+C\right)=0
$$

where

$$
\begin{aligned}
& B=\alpha+\gamma+\delta+\mu-\beta_{2}, \\
& C=\alpha\left(\gamma+\delta+\mu-\beta_{2}\right)-\kappa(\delta+\mu) \beta_{1} .
\end{aligned}
$$

Here, we have $\lambda_{1}=-\mu, \lambda_{2}=-(\theta+\mu)$, and other solutions are negative provided that $B$ and $C$ are positive, this is possible that when $R_{D}<1$.

$$
\begin{aligned}
B & =\alpha+\gamma+\delta+\mu-\beta_{2}>\gamma+\delta+\mu-\beta_{2} \\
& =\frac{\kappa(\delta+\mu) \beta_{1}+\alpha \beta_{2}}{\alpha R_{D}}-\beta_{2} \text { since } R_{D} \text { in }(18) \\
& =\frac{\kappa(\delta+\mu) \beta_{1}+\alpha \beta_{2}\left(1-R_{D}\right)}{\alpha R_{D}}>0, \text { for } R_{D}<1,
\end{aligned}
$$

$$
C=\alpha\left(\gamma+\delta+\mu-\beta_{2}\right)-\kappa(\delta+\mu) \beta_{1}=\alpha(\gamma+\delta+\mu)\left(1-R_{D}\right)
$$
$>0$, for $R_{D}<1$.

For $R_{D}=1$, the quadratic factor in equation (25) has one zero eigenvalue; it follows that the Jacobian matrix (24) has a simple zero eigenvalue and other eigenvalues are negative and the equilibrium point is nonhyperbolic at $R_{D}=1$. Linearization does not show the stability of nonhyperbolic equilibrium points, so we will analyze it using the central manifold theory $[29,30]$ stated below. 
Theorem 1. Consider the following general system of ordinary differential equations with a bifurcation parameter $\phi$,

$$
\frac{d x}{d t}=f(x, \phi), \quad f: \mathbb{R}^{n} \times \mathbb{R} \longrightarrow \mathbb{R}^{n}, f \in \mathbb{C}^{2}\left(\mathbb{R}^{n} \longrightarrow \mathbb{R}\right) .
$$

Without loss of generality, it is assumed that 0 is an equilibrium for system (28) for all values of the parameter $\phi$; that is, $f(0, \phi) \equiv 0$ for all $\phi$. Assume that

A1. $A=D_{x} f(0,0)=\left(\partial f_{i} / \partial x_{j}\right)(0,0)$ is the linearization matrix of system ((28)) around the equilibrium 0 with $\phi$ evaluated at 0 . Zero is a simple eigenvalue of $A$, and all other eigenvalues of $A$ have negative real parts;

A2. Matrix $A$ has a nonnegative right eigenvector $w$ and $a$ left eigenvector $v$ corresponding to the zero eigenvalue.

Let $f_{k}$ be the $k^{\text {th }}$ component of $f$ and

$$
\begin{aligned}
& a=\sum_{k, i, j=1}^{n} v_{k} w_{i} w_{j} \frac{\partial^{2} f_{k}}{\partial x_{i} \partial x_{j}}(0,0), \\
& b=\sum_{k, i, j=1}^{n} v_{k} w_{i} \frac{\partial^{2} f_{k}}{\partial x_{i} \partial x_{j}}(0,0) .
\end{aligned}
$$

The local dynamics of the ODE in ((28)) around 0 is totally determined by $a$ and $b$ given in ((29)).

(i) $a>0, b>0$. When $\phi<0$ with $|\phi|<<1,0$ is locally asymptotically stable, and there exists a positive unstable equilibrium; when $0<\phi<<1,0$ is unstable and there exists a negative and locally asymptotically stable equilibrium.

(ii) $a<0, b<0$. When $\phi<0$ with $|\phi|<<1,0$ is unstable; when $0<\phi<<1,0$ is locally asymptotically stable, and there exists a positive unstable equilibrium.

(iii) $a>0, b<0$. When $\phi<0$ with $|\phi|<<1,0$ is unstable, and there exists a locally asymptotically stable negative equilibrium; when $0<\phi<<1,0$ is stable, and a positive unstable equilibrium appears.

(iv) $a<0, b>0$. When $\phi$ changes from negative to positive, 0 changes its stability from stable to unstable. Correspondingly, a negative unstable equilibrium becomes positive and locally asymptotically stable.

Now setting $S=x_{1}, I=x_{2}, R=x_{3}$, and $D=x_{4}$, we can rewrite the system of model (2) as follows:

$$
\begin{aligned}
& \frac{d x_{1}}{d t}=\Lambda-\frac{\left(\kappa \beta_{1} x_{4}+\beta_{2} x_{2}\right) x_{1}}{x_{1}+x_{2}+x_{3}}-\mu x_{1}+\theta x_{3}:=f_{1}, \\
& \frac{d x_{2}}{d t}=\frac{\left(\kappa \beta_{1} x_{4}+\beta_{2} x_{2}\right) x_{1}}{x_{1}+x_{2}+x_{3}}-(\gamma+\delta+\mu) x_{2}:=f_{2}, \\
& \frac{d x_{3}}{d t}=\gamma x_{2}-(\theta+\mu) x_{3}: f_{3}, \\
& \frac{d x_{4}}{d t}=(\delta+\mu) x_{2}-\alpha x_{4}:=f_{4} .
\end{aligned}
$$

With $R_{D}=1$ corresponding to $\beta_{1}=\beta_{1}^{*}$ where

$$
\beta_{1}^{*}=\frac{\alpha(\gamma+\mu+\delta)-\alpha \beta_{2}}{\kappa(\delta+\mu)},
$$

which is assumed to be a bifurcation parameter. The diseasefree equilibrium is

$$
X^{0}=\left(x_{1}^{0}, x_{2}^{0}, x_{3}^{0}, x_{4}^{0}\right)=\left(\frac{\Lambda}{\mu}, 0,0,0\right) .
$$

The linearization matrix of system (30) around the disease-free equilibrium when $\beta_{1}=\beta_{1}^{*}$ is

$$
D_{X} f=\left(\begin{array}{cccc}
-\mu & -\beta_{2} & \theta & -\kappa \beta_{1}^{*} \\
0 & \beta_{2}-(\gamma+\mu+\delta) & 0 & \kappa \beta_{1}^{*} \\
0 & \gamma & -(\theta+\mu) & 0 \\
0 & \delta+\mu & 0 & \alpha
\end{array}\right) \text {. }
$$

It is clear that $D_{x} f$ has a simple zero eigenvalue and a right eigenvector corresponding to the zero eigenvalue is $w=\left(w_{1}, w_{2}, w_{3}, w_{4}\right)$, where

$$
\begin{aligned}
& w_{1}=\frac{(\gamma \mu+(\delta+\mu)(\mu+\theta)) \alpha}{\mu(\mu+\theta)}, \\
& w_{2}=\alpha, \\
& w_{3}=\frac{\alpha \gamma}{\mu+\theta}, \\
& w_{4}=\mu+\delta,
\end{aligned}
$$

and the left eigenvector associated with the zero eigenvalue satisfying $w \cdot v=1$ is $v=\left(v_{1}, v_{2}, v_{3}, v_{4}\right)$.

$$
\begin{aligned}
& v_{1}=0, \\
& v_{2}=\frac{1}{(\alpha+\gamma+\mu+\delta)-\beta_{2}}, \\
& v_{3}=0, \\
& v_{4}=\frac{1}{\delta+\mu} .
\end{aligned}
$$

Based on the theoretical result given in Theorem 1, we have to compute $a$ and $b$ at $\left(X^{0}, \beta_{1}^{*}\right)$ where

$$
\begin{aligned}
& a=\sum_{k, i, j=1}^{4} v_{k} w_{i} w_{j} \frac{\partial^{2} f_{k}}{\partial x_{i} \partial x_{j}}\left(X^{0}, \beta_{1}^{*}\right), \\
& b=\sum_{k, i=1}^{4} v_{k} w_{i} \frac{\partial^{2} f_{k}}{\partial x_{i} \partial \beta_{1}}\left(X^{0}, \beta_{1}^{*}\right) .
\end{aligned}
$$


Since $v_{1}=v_{3}=0$, equations $a$ and $b$ in (36) becomes

$$
\begin{aligned}
& a=v_{2} \sum_{i, j=1}^{4} w_{i} w_{j} \frac{\partial^{2} f_{2}}{\partial x_{i} \partial x_{j}}\left(X^{0}, \beta_{1}^{*}\right)+v_{4} \sum_{i, j=1}^{4} w_{i} w_{j} \frac{\partial^{2} f_{4}}{\partial x_{i} \partial x_{j}}\left(X^{0}, \beta_{1}^{*}\right), \\
& b=v_{2} \sum_{i=1}^{4} w_{i} w_{j} \frac{\partial^{2} f_{2}}{\partial x_{i} \partial \beta_{1}}\left(X^{0}, \beta_{1}^{*}\right)+v_{4} \sum_{i=1}^{4} w_{i} \frac{\partial^{2} f_{4}}{\partial x_{i} \partial \beta_{1}}\left(X^{0}, \beta_{1}^{*}\right) .
\end{aligned}
$$

Substituting the eigenvectors and the computed partial derivatives of the system (30) at $\left(X^{0}, \beta_{1}^{*}\right)$ in the formula for $a$ and $b$ in (37), after some algebraic computation, we get

$$
\begin{aligned}
& a=-\frac{2 \mu v_{2}\left(w_{2}+w_{3}\right)}{\Lambda}\left(w_{2} \beta_{2}+w_{4} \kappa \beta_{1}^{*}\right)<0, \\
& b=v_{2} w_{4} \kappa>0 .
\end{aligned}
$$

This indicates that at $\beta_{1}=\beta_{1}^{*}$ for $R_{D}=1$, the system exhibits a transcritical bifurcation (i.e., the disease-free equilibrium point changes its stability from locally stable for $R_{D}<1$ to unstable for $R_{D}>1$ ). Consequently, Theorem 1 is guaranteed for the following result.

Theorem 2. The system in (2) exhibits a forward bifurcation at $\beta_{1}=\beta_{1}^{*}\left(\right.$ for $\left.R_{D}=1\right)$ and a locally stable positive endemic equilibrium will appear whenever $R_{D}>1$ but near to unity.

\subsubsection{Global Stability of Disease-Free Equilibrium Point}

Proposition 2. The disease-free equilibrium point of the system given (2) is globally asymptotically stable if $R_{D}<1$.

Proof. Let $a$ be an arbitrary chosen positive constant. Consider a nonnegative definite function on the invariant set $\Omega$ as

$$
V=I+a D, \quad a \geq 0 .
$$

Taking the derivative of $V$ gives $\dot{V}=\dot{I}+a \dot{D} \Rightarrow \dot{V}=\left(\kappa \beta_{1} D+\right.$ $\left.\beta_{2} I\right) S / N-(\gamma+\delta+\mu) I+\alpha((\mu+\delta) I+\alpha D)$ that

Since $S(t) \leq N(t)$ for all $t \geq 0$ in the domain $\Omega$, it follows

$$
\begin{aligned}
\dot{V} & \leq \kappa \beta_{1} D+\beta_{2} I-(\gamma+\mu+\delta) I+a(\mu+\delta) I-a \alpha D \\
& =\left(\beta_{2}-(\gamma+\mu+\delta)+a(\mu+\delta)\right) I+\left(\kappa \beta_{1}-a \alpha\right) D .
\end{aligned}
$$

We can choose $a$ such that $\kappa \beta_{1}-a \alpha=0$; that is, $a=\kappa \beta_{1} / \alpha$

$$
\begin{aligned}
\Rightarrow \dot{V} & \leq\left(\beta_{2}-(\gamma+\mu+\delta)+\frac{\kappa \beta_{1}}{\alpha}(\mu+\delta)\right) I \\
& =(\gamma+\mu+\delta) I=(\gamma+\mu+\delta) I\left(\frac{\kappa(\delta+\mu) \beta_{1}+\alpha \beta_{2}}{\alpha(\gamma+\delta+\mu)}-1\right) \\
& =(\gamma+\mu+\delta) I\left(R_{D}-1\right) \leq 0, \text { for } \quad R_{D} \leq 1
\end{aligned}
$$

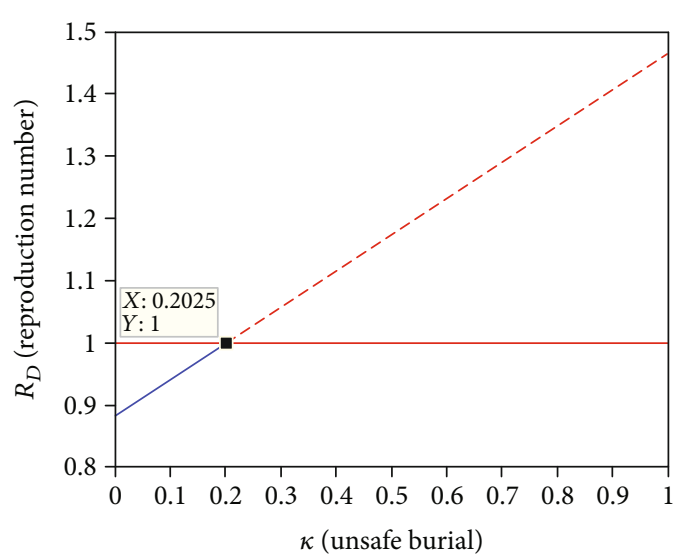

FIgURE 2: Graph of reproduction number $R_{D}$ as a function of $\kappa$. Other parameter values are given in Table 1.

Since all parameters and variables in the model (2) are nonnegative, the derivative of the Lyapunov function is $\dot{V}$ $\leq 0$ if $R_{D} \leq 1$ and $\dot{V}=0$ if and only if $I=0$. It follows that from LaSalle's invariance principle [31], the only invariant singleton set in $\Omega$ is the set $\left\{E^{0}\right\}$.

Hence, the disease-free equilibrium point is globally asymptotically stable for $R_{D}<1$ in the invariant region $\Omega$.

\section{Numerical Analysis}

In this section, some numerical simulations of model (2) are carried out for the data shown in Table 1.

The main objective is to understand and investigate the impact of unsafe handling $(\kappa)$ and disposition rate $(\alpha)$ of the dead bodies of infected individuals during Nipah outbreak. The proposed model is also compared with that of the SIR model where the dead body has no contribution towards transmission of infection. Using the reproduction number $R_{D}$ in (18) and solving $R_{D}=1$, the critical value of $\kappa$ is computed as 0.20249 , by assuming parameters as given in Table 1 with $\alpha=1$ day $^{-1}$. From $R_{D}$ versus $\kappa$ curve, drawn in Figure 2, it is observed that $R_{D}<1$ for $0 \leq \kappa<0.20249$ and $R_{D}>1$ for $0.20249<\kappa \leq 1$. It means that the high ratio of unsafe burial practice increases the reproduction number, and this is leading to instability of disease-free state (Proposition 1) and existence of endemic state (Lemma 2).

Furthermore, if we assume all dead body contacts are unprotected $(\kappa=1)$ and for our choice of data, we have got the critical value of $\alpha=4.9383$ day $^{-1}$. This is the value where the reproduction number is close to unity. As shown in Figure 3 , the reproduction number is inversely related with the rate of disposing dead bodies. For $\alpha>4.9383$ day $^{-1}$, the reproduction number is less than one and the infection decreases through time and finally dies out from the community.

Infection curves for various values of $\kappa$ with $\alpha=1$ day $^{-1}$ are drawn in Figure 4. For different values of $\kappa$ with $R_{D}>1$, the infection increases with time up to a peak point then decreases, converging to endemic level. For instance, when $\kappa=1$, the infection is sharply increasing up to a peak value 


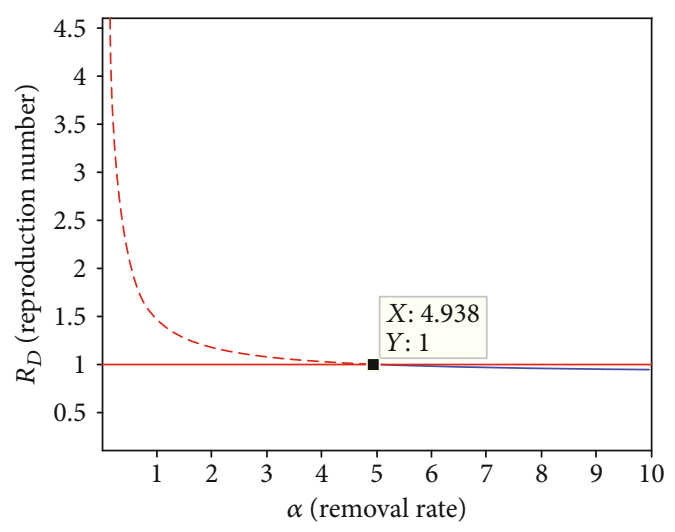

Figure 3: Graph of reproduction number $R_{D}$ as a function of $\alpha$. Other parameter values are given in Table 1.

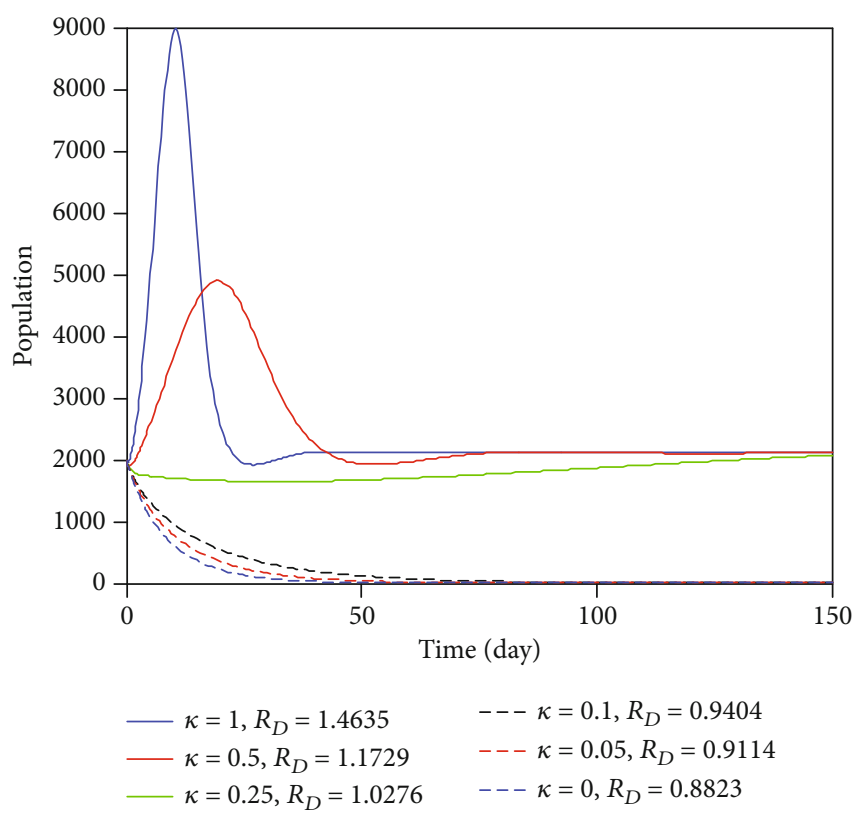

Figure 4: The dynamics of infectious population for $\alpha=1$ with different values of $\kappa$ and other parameter values are given in Table 1 .

and then decreasing to stabilize to the endemic equilibrium level $I^{*}=2107.5$. A similar pattern is observed for $\kappa=0.5$ and 0.25 , with $I^{*}=2107$ and $I^{*}=2103.5$, respectively. The peak is delayed and decreases with decreasing $\kappa$. It may be noted that there is a significant change in the peak values for different values of $\kappa$. The equilibrium level is also decreasing with decreasing $\kappa$ although not at the same rate as for the peak value. Thus, the increase in safe burial decreases the reproduction number which slows down the spread of infection and the peak of infection as well as the equilibrium level is also lowered.

Furthermore, the remaining values of $\kappa$ we used in the graph make the value of $R_{D}<1$. So, broken line curves in Figure 4 show the infection decreases and stabilizes at the disease-free state with time. When safe burial increases, the infection decreases faster and will die out from the community.

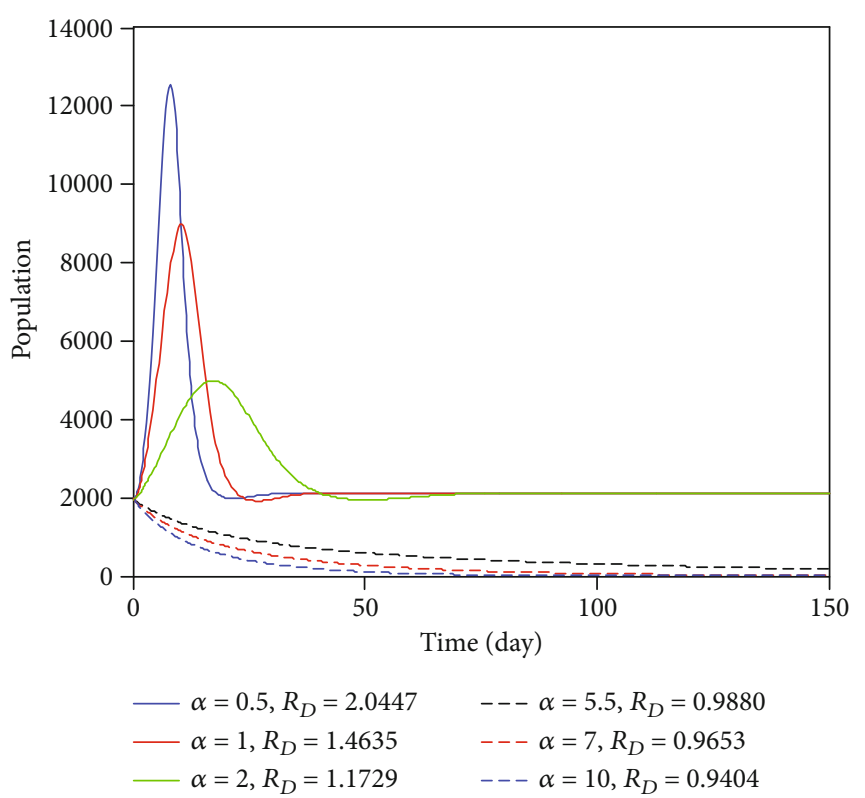

FIGURE 5: The dynamics of infectious population for $\kappa=1$ with different values of $\alpha$ and other parameter values are given in Table 1 .

In Figure 5, it is seen the infection curves for different values of $\alpha$ and assuming all contacts with the dead body are unprotected $(\kappa)$. As the rate of disposal $(\alpha)$ increases for $R_{D}>1$, the peak value of the infection decreases with time and finally comes to stabilize at endemic state. For $R_{D}<1$, the infection decreases fast and stabilize at disease-free state.

\section{Conclusion}

In most of human culture, there may be contact with the dead body during last rites due to bathing, expressing love or respect by hugging, touching heads and faces, etc. of the dead body particularly very close relatives. Researchers confirmed such contact of the dead body of Nipah-infected individuals is one way of transmitting disease from person to person. They recommend safe and protected contact for healthcare workers and family caregivers when handling the dead body. In this paper, we have proposed the SIRD model and presented the impact of unprotected contact with the dead bodies of Nipah-infected individuals and their disposal (burial or cremation) on the spread of Nipah infection. It is seen the numerical and analytical behavior of the dynamics that the high fraction of unprotected contact increases the reproduction number. It causes the disease to persist in the community and contributes some difficulty for the control of the outbreak. For the given choice of data, it is observed that the reproduction number $R_{D}$ is greater than one for $0.20249<\kappa \leq 1$. However, for $0 \leq \kappa<0.20249$, $R_{D}$ is less than one. For $\kappa=0$, the handling of the human dead body is well protected and no possibility of transmitting disease through it. In this case, the model is reduced to SIR.

Furthermore, the rate of disposal of dead bodies is inversely related with the reproduction number. In the absence of protected contact $(\kappa=1)$ and for the choice of data, $R_{D}$ is less than unity when $\alpha>4.9383$ day $^{-1}$ and greater 
than unity when $0<\alpha<4.9383 \mathrm{day}^{-1}$. If the handling of the human dead body to be buried is unprotected, it should be removed within a quarter of a day to minimize outbreak. This result shows that applying some effort to reduce unprotected contact and removing the dead body of infected individuals within a short period of time will make the reproduction number less than one and it helps in controlling the Nipah outbreak.

\section{Data Availability}

The parameter data used to support the findings of this study are included within the article.

\section{Conflicts of Interest}

The authors declare that there is no conflict of interest.

\section{References}

[1] K. B. Chua, W. J. Bellini, P. A. Rota et al., "Nipah virus: a recently emergent deadly paramyxovirus," Science, vol. 288, no. 5470, pp. 1432-1435, 2000.

[2] D. K. Chanchal, S. Alok, M. Sabharwal, R. K. Bijauliya, and S. Rashi, "Nipah: silently rising infection," International Journal of Pharmaceutical Sciences and Research, vol. 9, no. 8, pp. 3128-3135, 2018.

[3] WHO, "Surveillance and outbreak alert; nipah virus outbreaks in the who south-east asia region," $2018 \mathrm{http}$ ///origin.searo .who.int/entity/emerging_diseases/links/nipah_virus_ outbreaks_sear/en/.

[4] V. K. Chattu, R. Kumar, S. Kumary, F. Kajal, and J. K. David, "Nipah virus epidemic in southern india and emphasizing "one health" approach to ensure global health security," Journal of family medicine and primary care, vol. 7, no. 2, pp. 275-283, 2018.

[5] K. Halpin, A. D. Hyatt, R. Fogarty et al., "Pteropid bats are confirmed as the reservoir hosts of henipaviruses: a comprehensive experimental study of virus transmission," The American Journal of Tropical Medicine and Hygiene, vol. 85, no. 5, pp. 946-951, 2011.

[6] WHO, "Surveillance and outbreak alert; nipah virus-faqs," 2019 http://origin.searo.who.int/entity/en/.

[7] J. M. Montgomery, M. J. Hossain, E. Gurley et al., "Risk factors for nipah virus encephalitis in bangladesh," Emerging infectious diseases, vol. 14, no. 10, pp. 1526-1532, 2008.

[8] A. Chakraborty, H. M. S. Sazzad, M. J. Hossain et al., "Evolving epidemiology of nipah virus infection in Bangladesh: evidence from outbreaks during 2010-2011," Epidemiology \& Infection, vol. 144, no. 2, pp. 371-380, 2016.

[9] K. B. Chua, K. J. Goh, K. T. Wong et al., "Fatal encephalitis due to nipah virus among pigfarmers in Malaysia," The Lancet, vol. 354, no. 9186, pp. 1257-1259, 1999.

[10] N. I. Paton, Y. S. Leo, S. R. Zaki et al., "Outbreak of nipahvirus infection among abattoir workers in Singapore," The Lancet, vol. 354, no. 9186, pp. 1253-1256, 1999.

[11] E. S. Gurley, J. M. Montgomery, M. J. Hossain et al., "Personto-person transmission of Nipah virus in a Bangladeshi community," Emerging infectious diseases, vol. 13, no. 7, pp. 1031-1037, 2007.
[12] Y. Kaku, "Nipah virus infection," Nihon rinsho. Japanese journal of clinical medicine, vol. 65, p. 154, 2007.

[13] M. S. Chadha, J. A. Comer, L. Lowe et al., "Nipah virusassociated encephalitis outbreak, Siliguri, India," Emerging infectious diseases, vol. 12, no. 2, pp. 235-240, 2006.

[14] B. S. Ang, T. C. Lim, and L. Wang, "Nipah virus infection," Journal of Clinical Microbiology, vol. 56, no. 6, 2018.

[15] WHO, "Key facts; ebola virus disease," 2019 https://www.who .int/news-room/fact-sheets/detail/ebola-virus-disease.

[16] H. M. S. Sazzad, M. J. Hossain, E. S. Gurley et al., "Nipah virus infection outbreak with nosocomial and corpse-to-human transmission, bangladesh," Emerging infectious diseases, vol. 19, no. 2, pp. 210-217, 2013.

[17] WHO, "Who-guideline-for-management prevention-andcontrol-of-nipah-virus-infection," pp. 1-82, 2016, Available: http://www.moh.gov.bt/wp-content/uploads/afd-files/2014/ 11/WHO-guideline-for-Management-Prevention-and-Con .pdf.

[18] M. Biswas, "Model and control strategy of the deadly nipah virus (niv) infections in Bangladesh," Research \& Reviews in Biosciences, vol. 6, no. 12, pp. 370-377, 2012.

[19] M. H. A. Biswas, "Optimal control of nipah virus (niv) infections: a Bangladesh scenario," Journal of Pure and Applied Mathematics: Advances and Applications, vol. 12, no. 1, pp. 77-104, 2014.

[20] M. H. A. Biswas, M. M. Haque, and G. Duvvuru, "A mathematical model for understanding the spread of nipah fever epidemic in Bangladesh," in 2015 International Conference on Industrial Engineering and Operations Management (IEOM), pp. 1-8, IEEE, 2015.

[21] J. Sultana and C. N. Podder, "Mathematical analysis of nipah virus infections using optimal control theory," Journal of Applied Mathematics and Physics, vol. 4, no. 6, pp. 10991111, 2016.

[22] M. K. Mondal, M. Hanif, and M. H. A. Biswas, "A mathematical analysis for controlling the spread of nipah virus infection," International Journal of Modelling and Simulation, vol. 37, no. 3, pp. 185-197, 2017.

[23] N. H. Shah, A. H. Suthar, F. A. Thakkar, and M. H. Satia, "SEImodel for transmission of Nipah virus," The Journal of Mathematics and Computer Science, vol. 8, no. 6, pp. 714730, 2018.

[24] D. Sinha and A. Sinha, "Mathematical model of zoonotic nipah virus in south-east asia region," Acta Scientific Microbiology, vol. 2, no. 9, pp. 82-89, 2019.

[25] H. S. Nita, D. T. Niketa, A. T. Foram, and H. S. Moksha, "Control strategies for Nipah virus," International Journal of Applied Engineering Research, vol. 13, no. 21, pp. 1514915163, 2018.

[26] P. Agarwal and R. Singh, "Modelling of transmission dynamics of Nipah virus (niv): a fractional order approach," Physica A: Statistical Mechanics and its Applications, vol. 547, article 124243, 2020.

[27] H.-F. Huo and L.-X. Feng, "Global stability for an HIV/AIDS epidemic model with different latent stages and treatment," Applied Mathematical Modelling, vol. 37, no. 3, pp. 14801489, 2013.

[28] P. Van den Driessche and J. Watmough, "Reproduction numbers and sub-threshold endemic equilibria for compartmental models of disease transmission," Mathematical Biosciences, vol. 180, no. 1-2, pp. 29-48, 2002. 
[29] C. Castillo-Chavez and B. Song, "Dynamical models of tuberculosis and their applications," Mathematical Biosciences \& Engineering, vol. 1, no. 2, pp. 361-404, 2004.

[30] H. W. Berhe, O. D. Makinde, and D. M. Theuri, "Parameter estimation and sensitivity analysis of dysentery diarrhea epidemic model," Journal of Applied Mathematics, vol. 2019, Article ID 8465747, 13 pages, 2019.

[31] J. P. La Salle, The Stability of Dynamical Systems, SIAM, 1976. 